\title{
Biocompatibility of Biomaterials
}

\author{
Hemant Raut ${ }^{1}$, Rupambika Das ${ }^{1}$, Ziqian $_{\text {Liu }}^{2}$, Liu Xiaoling ${ }^{2}$, and Seeram Ramakrishna ${ }^{3}$ \\ ${ }^{1}$ Singapore University of Technology and Design \\ ${ }^{2}$ University of Nottingham - Ningbo China \\ ${ }^{3}$ National University of Singapore
}

April 28, 2020

\begin{abstract}
There is a vast number of biomaterials ranging from drug-eluting stents, coated implants, drug delivery devices and artificial organs, among others, that have been developed in recent years. However, translation of many of these biomaterials to clinic is often plagued by biocompatibility challenges. This review focuses on strategies implemented in some of the recently developed biomaterials - particularly for soft and hard tissue regeneration, organ manufacturing and disease remediation - to overcome potential foreign body response to the incorporation of the biomaterials in the host.
\end{abstract}

\section{Hosted file}

Final Draft Review_reducedFS.pdf available at https://authorea.com/users/310006/articles/440854biocompatibility-of-biomaterials 